\title{
Letter to editor for the article "The effect of short-term preoperative ureteral stenting on the outcomes of retrograde intrarenal surgery for renal stones"
}

\author{
Subhajit Mandal ${ }^{1}$. Sudheer K. Devana ${ }^{1}$ - Girdhar S. Bora ${ }^{1}$ \\ Received: 12 March 2019 / Accepted: 16 March 2019 / Published online: 23 March 2019 \\ ○) Springer-Verlag GmbH Germany, part of Springer Nature 2019
}

\section{Dear Editor,}

We read the article with great interest published in your esteemed journal titled "The effect of short-term preoperative ureteral stenting on the outcomes of retrograde intrarenal surgery for renal stones" by Min Ho Lee et al. We congratulate the authors for performing the study with the chief aim of assessing the effect of preoperative ureteric stenting duration on the outcomes of RIRS for renal stones. However, there are certain observations, which need to be reevaluated.

In the methodology section, the authors should have mentioned the rationale behind excluding patients with a preoperative stented period more than 30 days before undergoing RIRS. The authors should also mention whether the surgeons followed any specific reasons/criteria for considering intraoperative ureteral balloon dilation during RIRS such as difficulty in passing access sheath or narrow ureteric orifice, etc. Surgeons doing RIRS in group 1 might have intentionally done more of ureteric dilatation leading to falsely significant observation. Also what specific criteria were followed for use of UAS of different sizes and double $\mathrm{J}$ stent placement at the end of the procedure should be mentioned in the methodology.

In Table 1, the authors should also mention the proportions of patients needing the use of access sheath and double
$\mathrm{J}$ stent placement at the end of the procedure in all the three groups as these values might have bearing on the readmission rate and also on post-operative complication rate. Traxer et al. [1] had shown that use of ureteral access sheath maintains a lower intraoperative intrapelvic pressure which later translates into a lower incidence of sepsis. The distribution of use of access sheath in the present study might be a confounding factor leading to falsely increased incidence of infective complication in group 1 (7.9\%).

Hence, considering the above observations we opine that the final conclusion that preoperative stenting can prevent high-grade ureteral injuries and reduce the rate of intraoperative balloon dilatation should be taken with a pinch of salt.

\section{Reference}

1. De Coninck V, Keller EX, Rodríguez-Monsalve M, Audouin M, Doizi S, Traxe O (2018) Systematic review of ureteral access sheaths: facts and myths. BJU Int 122(6):959-969. https://doi. org/10.1111/bju.14389 (Epub 2018 Jun 22)

Publisher's Note Springer Nature remains neutral with regard to jurisdictional claims in published maps and institutional affiliations.
Subhajit Mandal

drsubhajitmandal89@gmail.com

1 Post Graduate Institute of Medical Education and Research Chandigarh, Chandigarh, India 